\title{
DEVELOPMENT OF A SCHEIMPFLUG SLIT LAMP CAMERA SYSTEM FOR QUANTITATIVE DENSITOMETRIC ANALYSIS
}

\author{
M. A. VIVINO ${ }^{1}$, S. CHINTALAGIRI ${ }^{2}$, B. TRUS $^{1}$ and M. DATILES ${ }^{2}$ \\ Bethesda, Maryland
}

\begin{abstract}
SUMMARY
We have developed a computerised video system for accurate quantification of lens opacities from Scheimpflug images. Using a modified Zeiss Scheimpflug camera and Macintosh computer, the system software can warn of improper exposure and can provide immediate results of cataract progression on individual patients. These results are obtained from a novel technique which automatically divides the lens into anatomical regions and computes regional densities in the standard optical density unit. Because of the system's speed, the operator can properly capture and analyse a cataract in seconds, compare results with past patient records and give feedback to the patient on the results of the analysis. The system provides the appropriate data integrity and formats for documenting and monitoring lens opacities. Such a system will be useful in longitudinal studies of cataracts (i.e. for natural history and anti-cataract drug trials).
\end{abstract}

Examination of published literature shows a variety of techniques have been employed for assessing and classifying the cataract. ${ }^{1-5}$ Slit lamps have been used since the early part of the twentieth century for observation of the lens. ${ }^{6,7}$ Additions and modifications have been made to the slip lamp over the years to enhance visualisation and provide better information content to the observer. Studies have shown that slit lamp data can be improved by polarising filters, ${ }^{8,9}$ or can be used in fluorescence studies. ${ }^{10} \mathrm{~A}$ recent slit lamp addition was described for measuring the optical quality of the lens. ${ }^{11}$ Prior to these developments, the optics of a slit lamp were modified, using Scheimpflug's principle, to produce a clear cross-sectional image of the lens. ${ }^{12-16}$ Development of the Scheimpflug to its full

From: 'Computer Systems Laboratory, Division of Computer Research and Technology, and ${ }^{2}$ Section on Cataract and Cornea, Ophthalmic Genetics and Clinical Services Branch, National Eye Institute, National Institutes of Health, US Dept. of Health and Human Services, Bethesda, Maryland, USA.

Correspondence to: M. Datiles, Bldg. 10, Room 10N226, NIH, Bethesda, MD 20892, USA. useful potential continues today. This paper does not explore yellowing, spectral issues or Scheimpflug optics but describes how a standard technique, borrowed from densitometry, spectrophotometry and electrophoresis, can be applied in computer-based quantification of opacities. The paper further describes a computer algorithm which divides the lens into anatomical regions.

In biological imaging, most notably in electrophoresis, sample concentrations can be determined using optical, electronic and computer-based techniques. The system front end is often a video imaging system, photodiode or other optical sensor. ${ }^{17}$ Densitometric science was described originally by Bouguer and Lambert, who described loss of radiation (or light) in passing through a medium. Later, Beer found that the radiation loss in a medium was a function of the substance's molarity or concentration. ${ }^{18}$ According to Beer's law, concentration is proportional to optical density (OD). It is our premise that increases in OD correlate directly with increases in cataracts. In order to evaluate this premise, there was a need for an instrument which could accurately measure OD values. This instrument would be of value in longitudinal cataract studies.

Modifications have been made to a Zeiss Scheimpflug slit lamp camera (SLC) so that it can be used for accurate quantitative densitometric analysis. We developed a new computer system to process the Zeiss SLC images and apply Beer's law to measure OD values. Various techniques, including computer models, have been reported for the analysis of Scheimpflug images. ${ }^{4,519-21}$ The lack of standardisation, especially with respect to units of measurement, and the technological limits associated with in vivo measurements, have been the primary limiting factors in objective methods for documenting cataracts numerically.

To use Beer's law with instrumentation often involves measurement of incident and transmitted light, or incident and reflected light. ${ }^{22}$ Our implementation of Beer's law uses the SLC video camera for lens opacity measurement. 


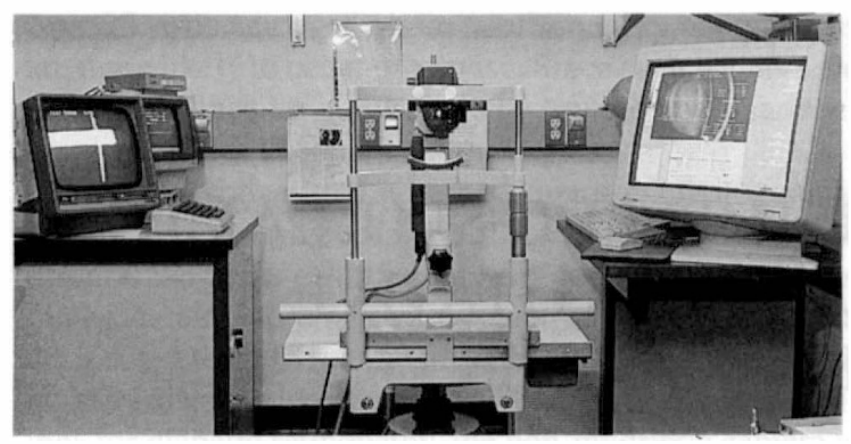

Fig. 1. The Scheimpflug system in use at NEI/NIH. The Zeiss Scheimpflug optics are seen in the centre. The large monitor on the right is part of the Macintosh Quadra. This is the system which does the image capture and analysis. The original Zeiss system is seen on the left.

The camera does not produce density readings directly, but captures a two-dimensional matrix of pixel values which are linear with respect to transmission. The video frame grabber transforms the video signal into pixel values between 0 and 255 ( 8 bits of quantisation). A calibration look-up table is then employed to convert pixel values to OD density using a technique involving standard neutral density filters and a curve-fitting procedure.

The original Zeiss Scheimpflug system did not contain built-in standards to use in converting pixel intensities to OD values. The optics, camera and lamp feedback electronics have been retained in a modified system. Hardware was developed to link the older Zeiss electronics to a Macintosh Quadra computer (Apple Computers Inc., 20525 Mariani Ave, Cupertino, CA 95014, USA). Software for imaging and cataract analysis has been developed on the Macintosh (Fig. 1).

The research and development objectives were a system for measuring cataracts which delivers an output in an accepted and standardised unit such as optical density, and a software system which performs image analysis in a user-friendly and systematic way. This software must allow easy but accurate comparison of longitudinal (follow-up) data.

\section{SIGNIFICANCE OF DATA}

The Zeiss system came with a Newvicon tube camera having a signal-to-noise ratio (SNR) of $55 \mathrm{~dB}-$ a figure high enough to give a true quantisation level of 8 significant bits. Cameras having lower SNRs are generally not used for medical or quantitative imaging purposes. Additionally, the video frame grabber must accurately convert the analogue video without loss of information. The Macintosh frame grabber used for these studies incorporates an 8-bit analogue to digital (A/D) converter with a differential non-linearity of less than one half of the least significant bit specified at the video bandwidth. ${ }^{23}$

\section{SYSTEM CALIBRATION}

\section{Density Calibration Technique}

We have developed a technique for imaging neutral density filters using a Zeiss-supplied integrating sphere.
This unique device was designed by Zeiss and sits at the focal point of the imaging optics (where the lens would be if a patient were under observation). Using the integrating sphere allows us to form a table of how neutral density filters attenuate light as received and passed through the Scheimpflug optics. Without the integrating sphere and filters, no standard exists for the Zeiss to correlate opacity with OD. When the operator initiates a lamp flash, a slit of light passes into a port on the integrating sphere. Light will then pass out from the port and into the receiving optics of the Scheimpflug instrumentation. Calibrated Kodak (Eastman Kodak Company, Rochester, NY 14650, USA) neutral density filters can be placed in the receiving optical path ahead of the video camera. Individual images of the sphere are acquired for measured filter densities of 0.09 to 1.33 and for no filter. Data obtained from the filter acquisition procedure will be used to calibrate patient data.

The technique described above introduces a small systematic error because no integrating sphere can achieve perfect reflectivity. However, the error is identical in all calibration images, and will amount to a small underestimate of values when calibration images are compared with images of the eye. The alternative technique is use of a beam splitter which will simultaneously pass light to the human eye as well as through a calibrated step wedge. The two optical paths can again form one image by aligning side-by-side on an image sensor. It was not practical to modify the Zeiss optical system in this way; however, the Topcon Scheimpflug has the advantage of a built-in wedge (Topcon Instrument Corporation, 65 West Century Road, Paramus, NJ 07652, USA). Our development project would have benefited if we could have obtained a system which combined video acquisition with a fine-grade, wide-dynamic-range step tablet in the optical path of the instrument.

To develop the premise that the quantity of light backscattered into the Scheimpflug is proportional to the opacification in the lens, we had to account for the manner in which the computer, physician and OD scale describe the data (Fig. 2). The principles which we describe in this paper allow the calibration of pixel intensity values into OD. The camera and frame grabber pixel values are linear with respect to transmission $(T)$, which is the anti-log of the negative of OD: $T=10^{-O D}$

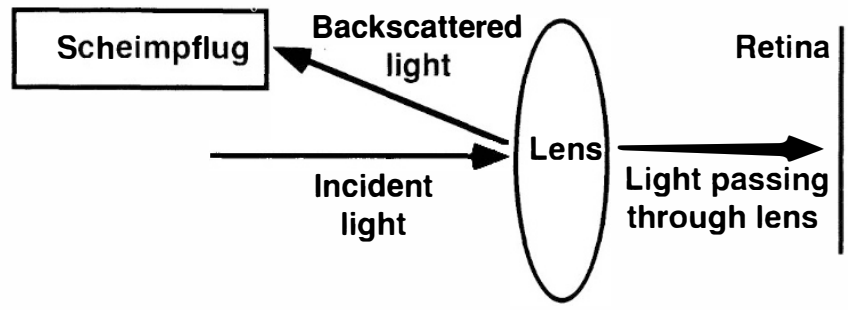

Fig. 2. The Scheimpflug measures backscattered light, although physicians refer to densities in terms of the light passing through the lens. Because of this, when calibrating Scheimpflug images to optical density the values for the optical density must be inverted. The diagram does not show the Scheimpflug instrument angle. 


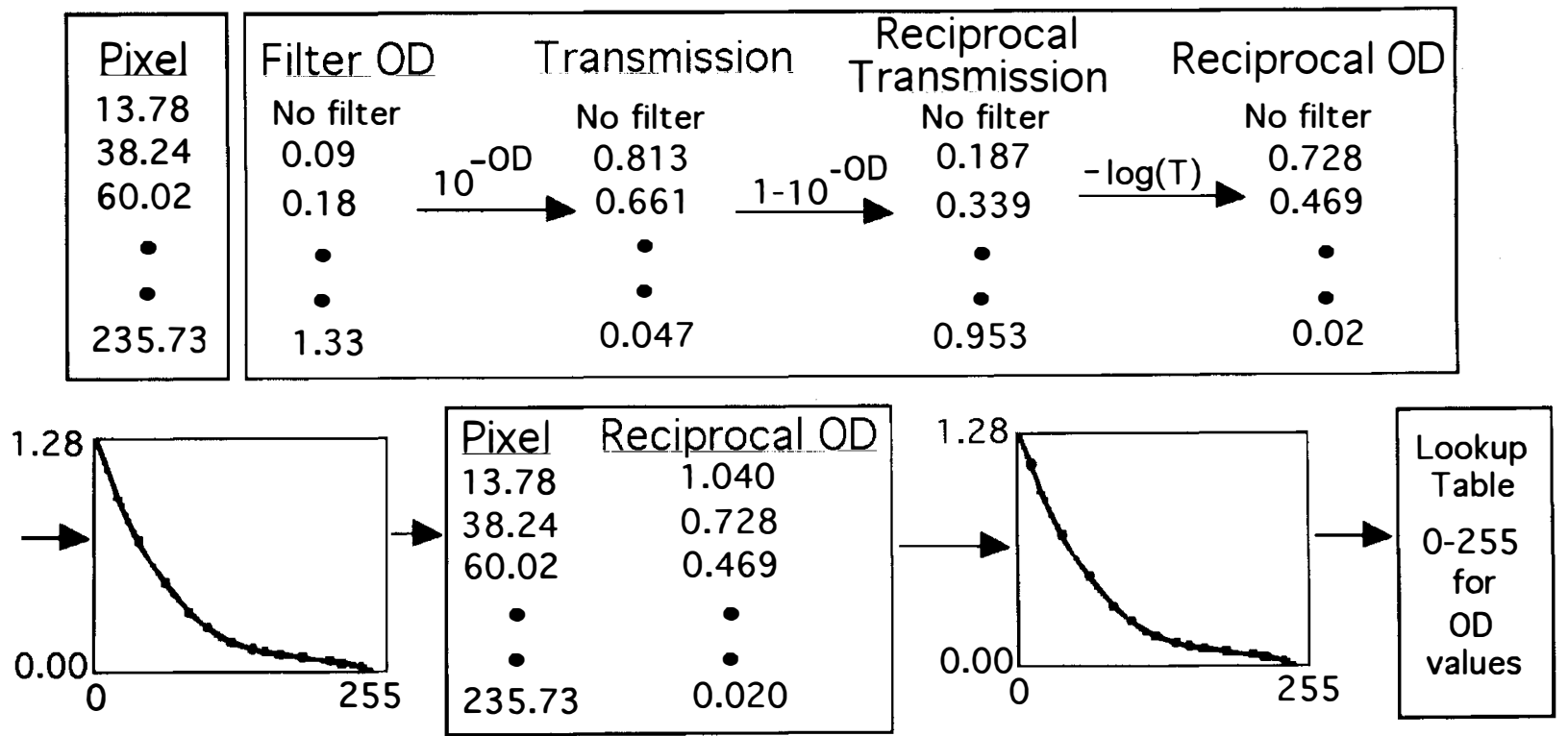

Fig. 3. The conversion of a pixel to optical density $(O D)$ requires several processing steps. The top left-hand box shows mean pixel values from images of the integrating sphere attenuated by filters. Adjacent to this box is the optical density inversion process. The two bottom left-hand boxes relate to obtaining a baseline density value for the 'no filter' case. The last two boxes show the final computation of a pixel to OD look-up table.

Since clinicians describe lens density in terms of light passing through the lens, and because we cannot measure this light with a Scheimpflug, a transformation is applied to calibration filter values to give their reciprocal OD. First note that $1-T$ is the remaining light which does not backscatter from the lens or does not pass through a neutral density calibration filter:

$$
A=1-T
$$

Next, by taking the minus log of the $1-T$ value, we can arrive at densities in clinically useful terms:

$$
\mathrm{OD}=-\log (A)
$$

Interested readers can refer to densitometric principles in various textbooks of physical and quantitative chemistry. ${ }^{18,24}$ The method and relationship to the human lens is best described by Weale in his discussion on lenticular light absorption. ${ }^{25}$ Please refer to Fig. 3, which shows a flow diagram of the entire pixel value to OD conversion process.

The Zeiss SLC has a useful feature of specific lamp voltage settings for image capture. The settings allow different illumination levels, permitting a wide spectrum of opacification levels to be seen and imaged properly by the instrumentation. To arrive at accurate density readings the video camera must not be operating in saturation or underexposure..$^{26}$ We have chosen 13 specific illumination levels to cover the range of patient data acquired. For each of the 13 intensity settings, 13 different neutral density calibration images are acquired. Using identical areas on each image, mean pixel values are then measured. Using the process described above and under Fig. 3, an exponential curve is used to fit pixel values into OD. This includes a baseline OD value determination for the image acquired containing no filter. The procedure is repeated for each lamp setting, producing 13 sets of calibration tables to correlate pixel intensities ( 0 to 255 ) with OD. The entire calibration procedure is repeated periodically to account for possible changes in the system over time.

\section{Spatial Calibration}

The system is spatially calibrated using a Zeiss-supplied standard grid. Each square of the grid corresponds to $0.5 \mathrm{~mm}$ distance. Images of the grid are acquired and measured. The spatial scale factor for our system is 1 pixel equals $0.01286 \mathrm{~mm}$.

\section{LENS ANALYSIS SOFTWARE}

The software was developed to produce an objective, systematic analysis of Scheimpflug images which also permits easy but accurate comparisons of longitudinal (follow-up) data. The system was designed to provide precise numerical information useful to a longitudinal study of cataract progression in the three anatomical zones: anterior, nuclear and posterior. To achieve these results in a clinical setting, software must automatically and reproducibly detect the centre of the lens as well as making the anatomical lens divisions: Cataract progression in various parts of the lens can be monitored separately with this technique. Scheimpflug images were obtained on patients with various types of cataracts, as well as normal volunteers. All patients and volunteers were participants in a clinical protocol approved by the intramural research board of the National Eye Institute and each gave full informed consent.

The results achieved with this system are dependent on the quality of acquired images. Since the entire analysis is performed in seconds while the patient is available, images can be recaptured if they fail to meet required standards. The entire analysis can be broken into a series of 


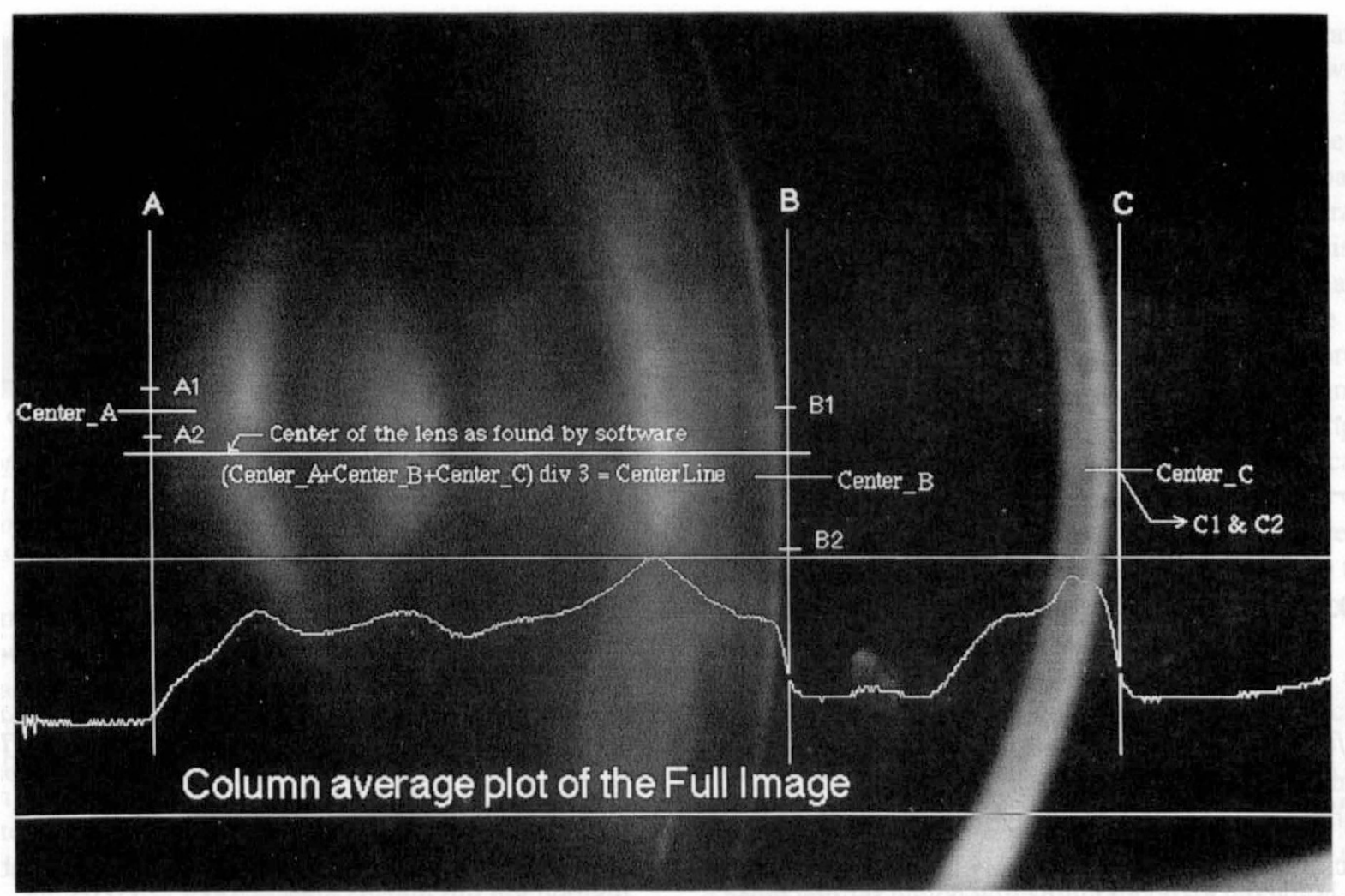

Fig. 4. A sample image superimposed with the column average plot of the entire image. The various options for the position of the centre of the lens have been depicted pictorially. A, column tangential to the posterior lens capsule; $B$, column tangential to the anterior lens capsule; $C$, column tangential to the corneal edge. Each tangential column has two extreme row's touching the column, depicted as $A 1, A 2, B 1, B 2, C 1$ and $C 2$.

sequential operations or protocols each of which is described in more detail below. All of the Scheimpflug analysis software was developed as our own addition to the NIH image program. The public domain NIH Image software was developed by Wayne Rasband of the National Institutes of Mental Health (e-mail address: wayne@helix.nih.gov).

\section{Locating the Centre of the Lens}

The apparent centre of the lens is found by first locating the vertical tangents to the edge of the cornea, edge of the anterior lens capsule and edge of the posterior lens capsule (C, B and $\mathrm{A}$ in Fig. 4). Vertical edge detection along columns A, B and C identifies the pairs of extreme rows touching these vertical tangents. These are seen and noted in Fig. 4 as points $\mathrm{A} 1$ and $\mathrm{A} 2$ on line $\mathrm{A}$, points $\mathrm{B} 1$ and $\mathrm{B} 2$ on line $\mathrm{B}$, and points $\mathrm{C} 1$ and $\mathrm{C} 2$ on line $\mathrm{C}$. For the example shown, $\mathrm{C} 1$ and $\mathrm{C} 2$ fall in the same position. The mean for each pair is computed as Centre_A, Centre_B and Centre_C. The mean of the three centre rows is computed as the default apparent centre line. The accuracy of the point determinations was based upon the ability of the software to choose the same point consistently during software development and criteria for picking the points were determined by trial and error by comparison with physician identification only. An operator option to override the computer permits use of Centre_A, Centre_B or Centre_C as the identified centre. Using all three positions as a decision basis allows the system to account for tilts existing in the image.

\section{Exposure Checking}

For accurate densitometry the lens must be imaged without any saturation or underexposure of the camera. The optimal lamp intensity level for each lens is selected to meet this criterion. Using the centre of the lens as the middle, a $0.6437 \mathrm{~mm}$ (50 pixel) vertical region of interest (ROI) is drawn through the entire lens. This ROI is slightly larger (in height) than the ROI that will be used during final analysis of posterior, nuclear and anterior density levels. Density levels within the ROI must fall within the bounds of the calibration filters imaged at that intensity setting (refer to Fig. 5). The range of density levels for each lamp setting is determined during calibration procedures, and is limited to density values in non-saturated or underexposed integrating sphere images. The setting at which the maximum percentage of pixels are in range (generally above $99 \%$ ) is considered to be the most suitable setting.

Exposure checking is performed immediately after image capture. This provides the clinician/operator with a mechanism to change the lamp intensity and recapture if necessary. There are cases where the image will remain underexposed (a young normal) or overexposed (advanced cataract) at all lamp settings. This occurs due to the limited dynamic range of the camera. In cases with out-ofrange levels, pixels can be truncated to a minimum or maximum allowable. However, a note is made in the patient database so that the images that have a high percentage $(>5 \%)$ of out-of-range pixels are not included in longitudinal studies. 


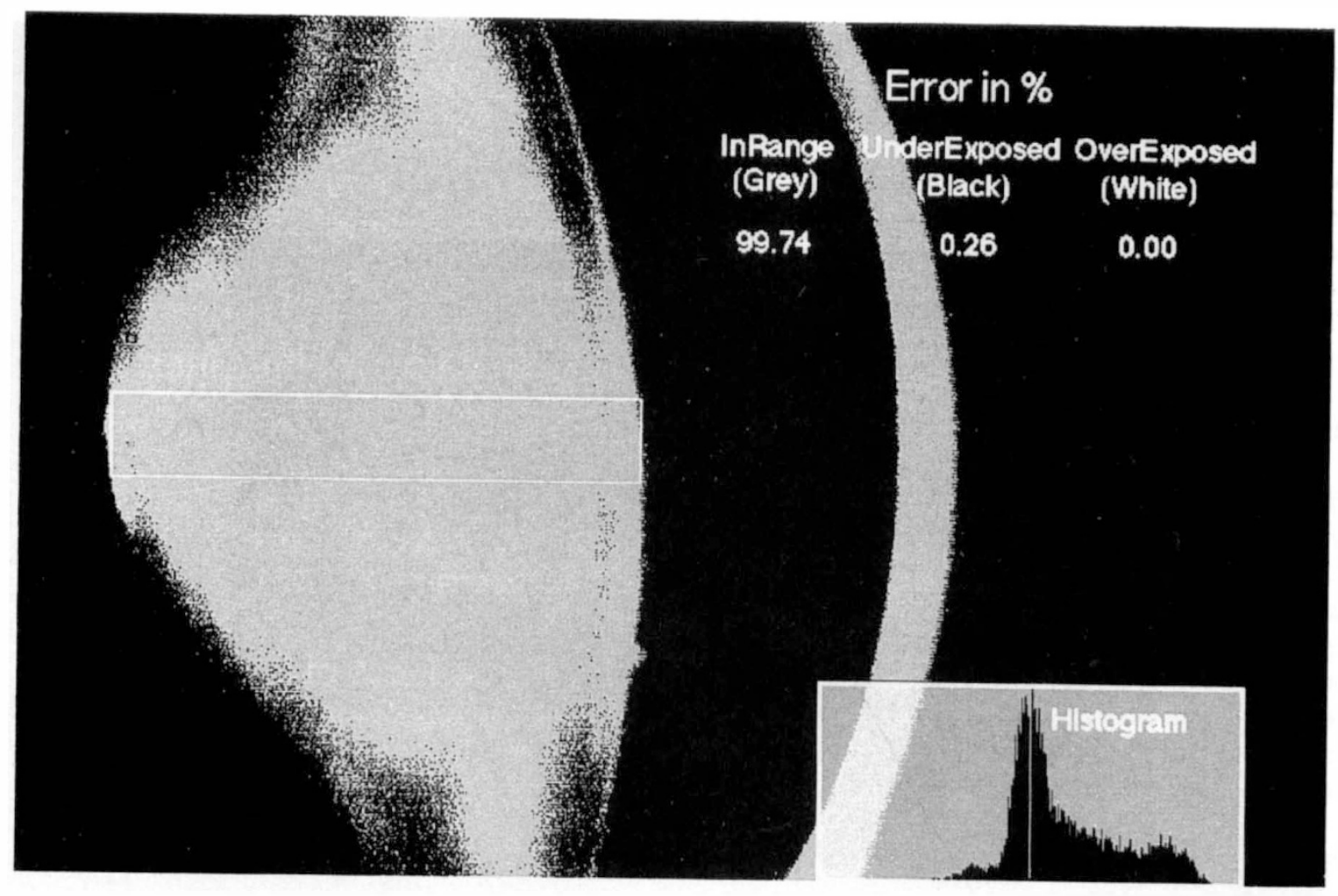

Fig. 5. Instantaneous feedback as displayed to the operator after image capture. Characteristics of the optimal lamp setting would include: (1) a histogram near the centre of the total operating range; (2) percentage of pixels which are free from any saturation or overexposure close to $100 \%$. For the region of interest shown, $99.74 \%$ of the pixels are in the proper camera range.

\section{Detection of the Posterior and Anterior Capsular Edges}

The posterior and anterior capsular edges must be found accurately because of the great density differences between the anterior chamber and lens, as well as the vitreous body and lens. If pixels from the anterior chamber or vitreous body are included, mean regional density values could be significantly affected. To avoid these pixels, a ROI $0.41 \mathrm{~mm}$ (32 pixels) high is created. The ROI encloses the central axis and runs the width of the lens. Repeated testing found the $0.41 \mathrm{~mm}$ height matched the lens curvature and avoided the external pixels. The search for edges involves finding the point of substantial slope increase in the average of each ROI column. Noise will not have substantial slope due to its uniform distribution and will be suppressed by column averaging. The search starts outside the lens and goes inwards, avoiding the possibility of detecting a cataract as the edge. Additionally, edge reflections in intense cataracts are discovered by locating a mid-sized gradient adjacent to a larger gradient. Software detects this combination and moves the edge appropriately. Alternative edge options are displayed when operator interaction indicates an improper edge.

\section{Detection of the Intralenticular Boundaries}

The algorithm to detect nucleus to posterior and nucleus to anterior boundaries took advantage of an observed characteristic pattern. A distinct local minimum at each region boundary was observed within the one-dimensional array derived from the average of each ROI column. Lenticular boundaries lie at the negative trough of each local minimum. Unfortunately, lens opacities create additional local minima which added to the complexity of software development.

The following operations are performed for each boundary detected: locate up to three minima, determine local density to see whether an advanced cataract exists, and provide positional boundary refinements if required. Using a set of rules, the algorithms pick the optimal boundaries from the available choices. The program will then display the remaining choices as options. Tests of these algorithms have been performed and have shown the decision logic to be reliable and accurate.

\section{Result Calculations}

The software displays the primary selection for the edges, intralenticular boundaries and the centre. If the clinician/ operator is not satisfied with the position for any of these, all the available options for the position are displayed and a suitable one may be selected interactively. If none of the options is acceptable, the operator may select the position manually using the mouse.

Once the operator is satisfied with the positions, the system proceeds with the analysis of the image. An ROI $0.41 \mathrm{~mm}$ ( 32 pixels) high, covering each region, is automatically chosen by the computer. The mean (calibrated OD units) and width $(\mathrm{mm})$ are computed for each region. The patient's clinical LOCS II grading score is then acquired interactively from the physician. ${ }^{3}$ This information may be used in comparative studies. No 


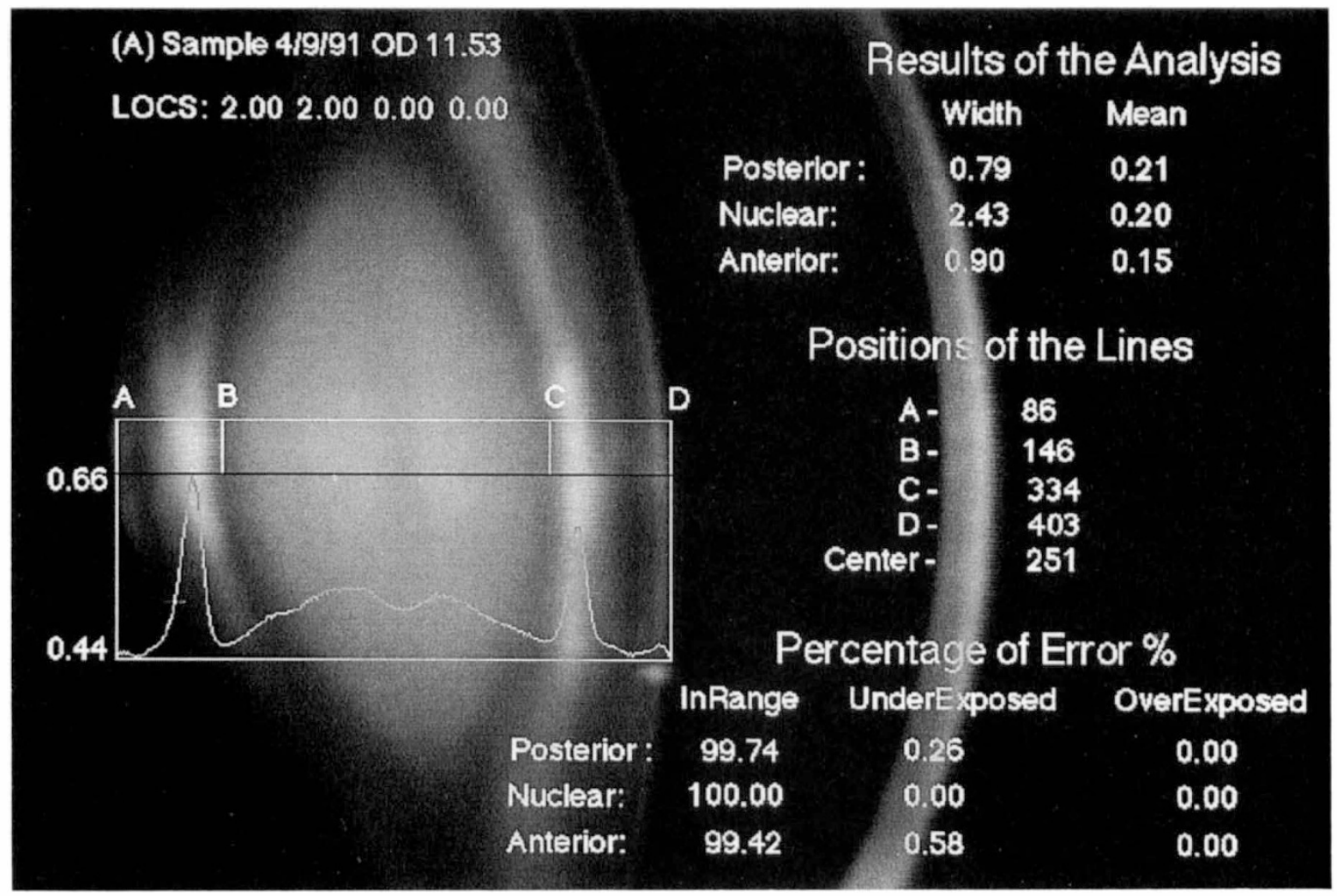

Fig. 6. An example report seen by the physician seconds after image capture. The data pertaining to the plot are the data used to detect each region. Final regional mean values are at the top right.

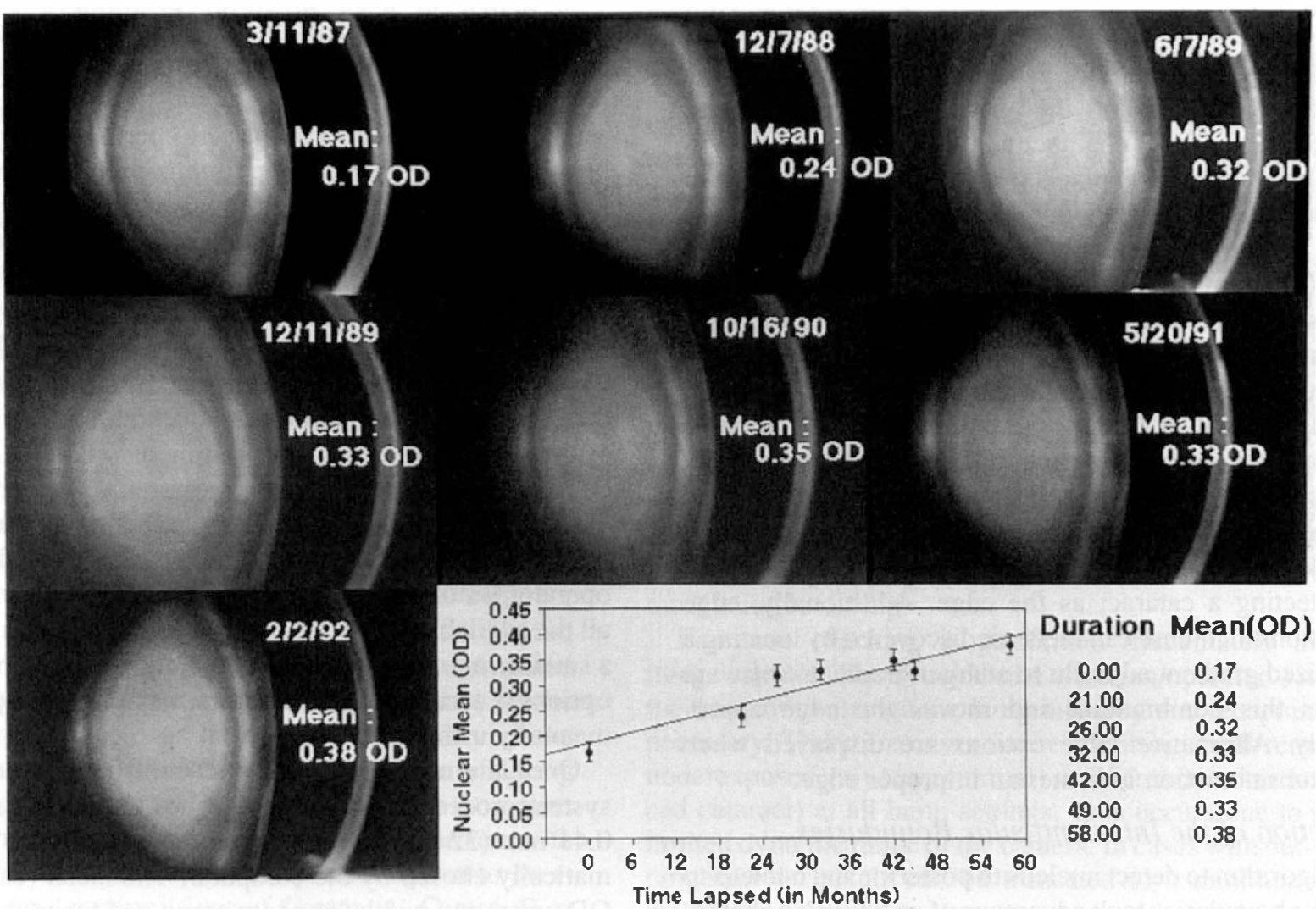

Fig. 7. A follow-up study of one patient covering 58 months. The study shows cataract progression in the nuclear region. Note the increase in mean optical densities for this patient's nuclear region. Calibration of images to optical density allows study of these images, despite the variation in their appearance. 
compensation is made to the data for densities due to natural age-related yellowing as opposed to densities associated with cataracts. Several studies of age-related densities show promise for developing a system which subtracts, or applies a function for subtracting, the natural yellowing densities. ${ }^{27,28}$ As the Scheimpflug develops, and as data on age-related densities are agreed upon, this feature could be included in future software updates. A sample analysed image with results is shown in Fig. 6.

\section{Output Files}

Multiple output files are created at the end of each analysis. One file contains information on the image just analysed and is used for defining analysis boundaries at a later date. A master file is updated to store the current analysis and to allow longitudinal and cross-sectional studies of prior images. To view the longitudinal data for the patient, provision is made for the operator to select text files created during earlier visits of the same patient. The system then generates a text file containing the time elapsed since the patient's first visit (in months) along with regional means (in OD units) for each visit. A progression chart can then be generated for the patient (Fig. 7).

\section{CONCLUSION}

The Scheimpflug system developed for use in the National Eye Institute possesses a variety of desirable features. The most important of these is the ability to calibrate images accurately into optical density units. Use of the optical density unit provides accurate quantification of opacities. The ability to ensure correct video exposure provides physicians with the highest quality and integrity of data possible. Finally, the technique developed to automatically subdivide the lens into clinical regions is important for quick interactive processing and comparison of patient data. These techniques will greatly facilitate comparison of longitudinal data (visit-to-visit) for each lens region (nucleus, anterior cortex, posterior cortex). Repeatability studies and detailed descriptions of the software are being documented and will be presented in the future.

\section{APPENDIX: FLOW LISTING FOR LENS EDGE/BOUNDARY DETECTION}

\section{-Abbreviation Explanation}

CA Column averages: an array which contains the average value for each column in the ROI it is generated from.

BPC Brightest pixel in column: an array which contains the brightest pixel value for each column in the ROI it is generated from.

FOG First order gradient: an array which corresponds to the computed gradient along an array of data. Computed as $-1,0,1$ along the data.

LOSP Loss of slope position.

OD Optical density

ROI Region of interest: an area of an image selected for analysis.
1. Locate the centre of the lens.

1. Compute CA on full image. 2. Locate centre of image. 3. Define critical search region. 4. Find the BPC. 5. Compute gradient array as CA-BPC. 6. Find columns $\mathrm{A}, \mathrm{B}$ and $\mathrm{C}$ using $1 \mathrm{D}$ edge detection along gradient. 7. For $\mathrm{A}, \mathrm{B}$ and $\mathrm{C}$ use $1 \mathrm{D}$ vertical detection to find $\mathrm{A} 1, \mathrm{~A} 2, \mathrm{~B} 1, \mathrm{~B} 2, \mathrm{C} 1$ and $\mathrm{C} 2$. 8. Find means as Centre _A of A1 and A2, Centre _B of B1 and B2, Centre $-C$ of $\mathrm{C} 1$ and $C 2$. 9. Find the mean of Centre _A, Centre_B, Centre_C. 10. Display to physician, allow for override.

2. Exposure checking or pre-analysis. Re-shoot the image if necessary and re-perform 1.

1. Autodraw $0.6437 \mathrm{~mm}$ vertical ROI through entire lens. 2. Check density levels to linear camera range table. 3. Inform physician if need to recapture.

3. Detection of the posterior and anterior capsular edges. 1. Autodraw $0.41 \mathrm{~mm}$ ROI surrounding centre of lens 2. Find CA for the $0.41 \mathrm{~mm}$ region. 3. Locate points of substantial slope increase in CA. 4. Build absolute value FOG along CA. 5. Check for reflection as small gradient adjacent to large gradient. 6. Display selection and optional choices to physician.

4. Detection of the intralenticular boundaries (nucleus to posterior and nucleus to anterior).

4.1. Determine operating environmental factors.

1. Create a small 'test' ROI in each clinical region and calculate the mean OD. 2. Define the search region (A to B) as an area to find the posterior to nuclear boundary. 3. Define the search region (C to $D$ ) as an area to find the anterior to nuclear boundary.

4.2. Calculate region statistics (minima, maxima, gradient).

1. Find the minimum and maximum for each critical region. 2. Find the maximum gradient position in each critical region.

4.3. Readjustment of local minima for consecutive adjacent minima.

1. Compare each column minimum found in step 4.2 with $\mathrm{CA}$ values from +10 to -10 columns away. See whether any value is within one (+ or - ) of the column minima. 2. If any of these 'flat regions' exist, find the midpoint as the new column minimum.

4.4. Readjustment of local minima in cases with loss of slope position (LOSP).

1. Check FOG for any combination of three consecutive ones and/or zeros (LOSP). 2. Use counter to count LOSPs in the top one-third and positions with two, one and/or zero combination. 3. After one-third of way past start of critical region locate a LOSP. If counter contains less than three counts, flag a LOSP for adjustment of the minima.

4.5. Pick the optimal inter-region boundaries from choices identified in past steps.

1. If no possible boundaries are found, consider the maximum gradient position within the critical region as the primary boundary position. 2 . If only one boundary is present, it is the primary boundary position. 3. If 
there are two boundary options and first option is detected very close to the start of the critical region $(<10$ pixels), ignore this first boundary possibility. The second boundary is the primary boundary position. 4. If there are three boundary options, the first possible boundary site in the direction of the search is always ignored. 5. If this point has been reached then two possible boundary sites exist. Physicians often identify a LOSP as a boundary. If one of the two remaining sites is a LOSP, it is chosen as the primary boundary. 6 . If two possible boundaries still remain, the operating environmental factors are examined for the final boundary determination. As a general rule, the boundary which is spatially furthest from any lower opacity region becomes the primary boundary selection. However, in cases when the two possibilities are both of a low opacity level, the distance to low opacities is not checked. In these cases, the least minimum is the final boundary choice.

5. Calculation of mean (OD) and width $(\mathrm{mm})$ for each region.

6. Save results.

The authors wish to thank the many physicians at NEI who both use and provide the feedback for improvements in the development of the Scheimpflug system. This includes Dr Benjamin V. Magno and Dr Susan M. Lasa. Thanks also to Wayne Rasband, whose publicly available Image program proved a rich source for development of the Scheimpflug software.

Key words: Cataract, Densitometry, Image processing, Ophthalmic instrumentation, Scheimpflug.

\section{REFERENCES}

1. Brown NAP, Bron AJ, et al. The objective assessment of cataract. Eye 1987;1:234-46.

2. Chylack LT, Leske MC, et al. Lens Opacities Classification System II (LOCS II). Arch Ophthalmol 1989;107:991-7.

3. Chylack LT, Wolfe JK, et al. The Lens Opacities Classification System, version III (LOCS III). Proceedings of the Sixth Congress of the US-Japan Cooperative Cataract Research Group (CCRG), 1991:170.

4. Edwards P, Datiles M, et al. Computerised cataract detection and classification. Curr Eye Res 1990;9:517-24.

5. Datiles MB, Edwards PA, Trus BL, Green SB. In vivo studies on cataracts using the Scheimpflug slit lamp camera. Invest Ophthalmol Vis Sci 1987;28:1707-10.

6. Tate G, Safir A. The slit lamp: history, principles and practice. In: Duane TD, editor. Clinical ophthalmology, vol I. Philadelphia: Lippincott, 1988:chapter 59.
7. Miller D. Optics and refraction. In: Podos S, Yanoff M, editors. Textbook of ophthalmology, vol 1. New York: Gower Medical, 1991: chapter 14.

8. Weale RA. New method for visualising discontinuities in the crystalline lens. Br J Ophthalmol 1986;70:925-30.

9. Weale RA. A further note on the photography of cataracts. Graefes Arch Clin Exp Ophthalmol 1988;226:468-70.

10. Lohmann W, Schmehl W, et al. Device for measuring the native fluorescence of lenses. J Biochem Biophys Methods 1988; $17: 155-8$.

11. Weale R. The Oqual: a new device for measuring the optical quality of the anterior segment of the human eye. Exp Eye Res 1992;55:507-10.

12. Drews RC. Depth of field in slit lamp photography. Ophthalmologica 1964;148:143-50.

13. Niesel $P$ von. Spalthampenphographie mit der Haag-StreitSpaltlampe 900. Ophthalmologica 1966;151:489-504.

14. Brown N. Slit-image photography. Trans Ophthalmol Soc 1969;89:397-408.

15. Brown N. An advanced slit-image camera. Br J Ophthalmol 1972;56:624-31.

16. Dragomirescu V, Hockwin O, et al. Development of a new equipment for rotating slit image photography according to Scheimpflug's principle. Interdisciplinary Topics Gerontol 1978;13:118-30.

17. Webster JG. Medical instrumentation: application and design. Boston: Houghton Mifflin, 1978:95,518-9.

18. Waser J. Quantitative chemistry. New York: Benjamin, 1964:229.

19. Laser H, Hannen K, Wienert J, Hockwin O. A new method for an objective classification of lens opacities. Dev Ophthalmol 1989;17:145-51.

20. Hockwin O, Dragomirescu V, et al. Measuring lens transparency by Scheimpflug photography of the anterior eye segment: principle, instrumentation, and application to clinical and experimental ophthalmology. J Toxicol Cutaneous Ocular Toxicol 1987;6:251-71.

21. Sparrow J, Brown N, et al. The Oxford modular cataract image analysis system. Eye 1990;4:638-48.

22. Kodak Corporation. Scientific imaging with KODAK films and plates. Eastman Kodak, 1987:9.

23. Micro Power Systems. Micro Power Systems for data acquisition and conversion ICs. 1992:3-79.

24. Findlay A, Kitchener JA. Practical physical chemistry. London: Longmans, Green, 1965.

25 . Weale R. The senescence of human vision. Oxford: Oxford University Press, 1992:88-9.

26. Spring KR, Lowy RJ. Characteristics of low light level television cameras. Methods Cell Biol 1989;29:269-89.

27. Weale R. The senescence of human vision. Oxford: Oxford University Press, 1992:90-2.

28. Kashima K, Trus BL, et al. Aging studies on normal lens using the Scheimpflug slit-lamp camera. Invest Ophthalmol Vis Sci 1993;34:263-9. 